\title{
Propagation of Spin Waves in Ferrite Films with Metasurface
}

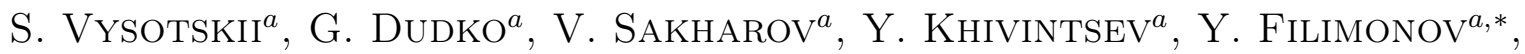 \\ N. Novitskil ${ }^{b}$, A. Stognij ${ }^{b}$ And S. Nikitov ${ }^{c}$ \\ ${ }^{a}$ Kotelnikov IRE RAS, Saratov Branch, 38 Zelenaya str., 410019, Saratov, Russia \\ ${ }^{b}$ SPMRC Natioanal Academy of Sciences of Belarus, 66 Independence Avenue, 220072, Minsk, Belarus \\ ${ }^{c}$ Kotelnikov IRE RAS, 11 Mokhovaya str., 125009, Moscow, Russia
}

\begin{abstract}
Magnetostatic surface waves (MSSW) propagation in YIG films with "leaky" and "resonant" metasurfaces (MS) is studied. "Leaky" MS was fabricated by ion etching of the subwavelength periodic structure in YIG film surface while "resonant" MS was formed by placing the subwavelength periodic array of YIG stripes onto the plane YIG film. The effects of filtration and the dispersion properties of MSSW in such structures are discussed.
\end{abstract}

DOI: 10.12693/APhysPolA.133.508

PACS/topics: 75.40.Gb, 75.25.-j, 75.30.Ds, 75.40.Mg

\section{Introduction}

Metasurface (MS) can be described as a twodimensional counterpart of metamaterial that consists of structural elements having subwavelength size as compared with the wavelength $\lambda$ of the incident wave [1, $2]$. Such structures are widely studied for possible application in controlling the propagation of electromagnetic and acoustic waves [1, 2]. Meanwhile, for the periodic magnetic structures, called also magnonic crystals (MC), the main interest, so far, was concentrated on investigation of propagation of spin waves with $\lambda$ comparable with the structure period $\Lambda$ because main attention was focused on the Bragg resonances appearing when $\Lambda=n \lambda / 2$ $(n=1,2,3, \ldots)$. The purpose of this paper is to study the propagation of magnetostatic surface waves (MSSW) in yttrium iron garnet (YIG) films with a MS $(\Lambda \ll \lambda)$ formed either by etching a subwavelength periodic structure in the films' surface (that could be called as "leaky MS") or by stacking a subwavelength periodic lattice of magnetic stripes and the plane YIG film (referred as "resonant MS").

\section{Experimental results and discussion}

Experiments were performed in the delay line (DL) configuration similar to one used in [3]. External inplane magnetic field $\boldsymbol{H}$ in the range of $0.1-1 \mathrm{kOe}$ was applied along the antennas, so its direction was always perpendicular to the wave vector $\boldsymbol{q}$ of emitted spin waves (SW) and corresponded to the Damon-Eshbach type of the wave or in other words MSSW. The vector network analyzer Agilent E5071C-480 was used to measure transmission $S_{21}(f)$ and reflection $S_{11}(f)$ characteristics of the DL in the frequency range $f=1-8 \mathrm{GHz}$ at different angles $\theta$ between the wave vector $\boldsymbol{Q}$ of Bragg lattice (perpendicular to the grooves direction) and $\boldsymbol{H}$, see Fig. 1.

*corresponding author; e-mail: yuri.a.filimonov@gmail.com

\subsection{Leaky $M S$}

The experiments were performed with structures based on the epitaxial YIG film with thickness $d \approx 7.7 \mu \mathrm{m}$, saturation magnetization $4 \pi M \approx 1750 \mathrm{G}$ and ferromagnetic resonance linewidth $2 \Delta H \approx 0.5$ Oe. The periodic lattice in the form of grooves with width of $4 \mu \mathrm{m}$, depth of $1 \mu \mathrm{m}$ and period $\Lambda \approx 7 \mu \mathrm{m}$ (see Fig. 1) was formed in the film surface using photolithography and ion etching.

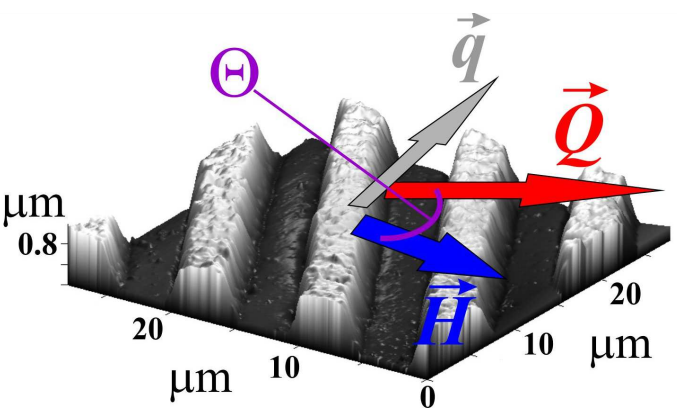

Fig. 1. Atomic-force microscopy image of the YIG film with leaky metasurface and geometry of the problem.

The shape anisotropy of the decorated surface leads to dynamical pinning of surface spins and effective hybridization of MSSW with the leaky exchange spin waves [3]. Such YIG film with a subwavelength surface structure by analogy with [1] can be considered as an example of ferromagnetic film with a "leaky MS". Under the conditions that the exchange waves are capable to perform multiple propagation through the film thickness $d$ and to be in "synchronism" with the surface structure period $\Lambda$, the MSSW losses increase resonantly and anomalous regions appear in the MSSW dispersion, see Figs. $2 \mathrm{a}, \mathrm{b}$ and $3 \mathrm{a}, \mathrm{b}$.

Formation of such resonances is caused by excitation of exchange guided modes and is possible in YIG films with microstructured surface only if the free path length $L$ of exchange SW exceeds both the thickness $d$ and the period A. Considered explanation was proved by micromagnetic 

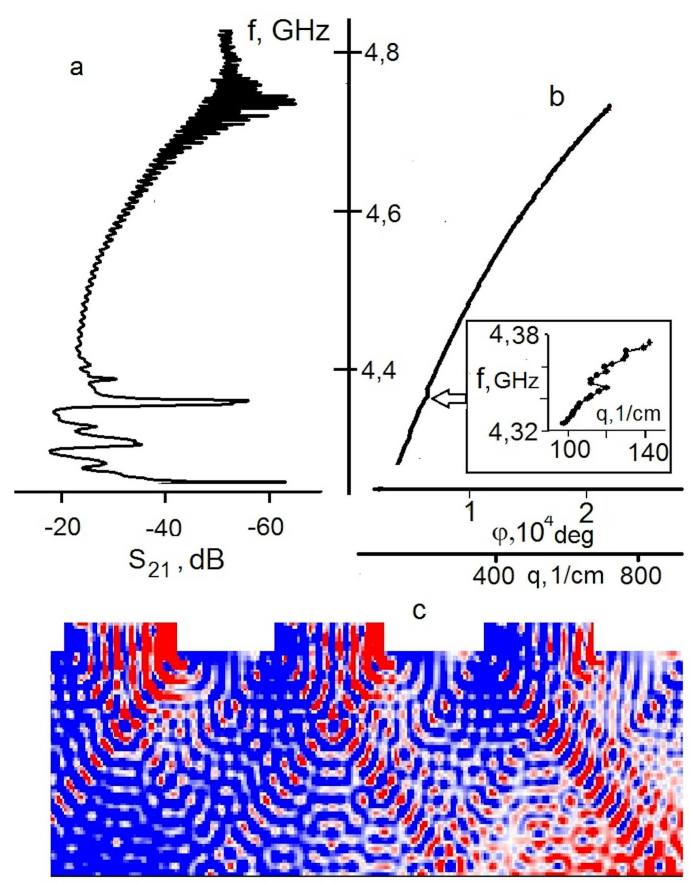

Fig. 2. Collinear Bragg geometry, applied field $H \approx$ 820 Oe corresponds to $\theta \approx 90^{\circ}$, MSSW propagates perpendicular to the grooves in MC with period $\Lambda \approx$ $7 \mu \mathrm{m}, d \approx 7.7 \mu \mathrm{m}$ : (a) MSSW transmission coefficients $S_{21}(f)$; (b) MSSW dispersion $f(q)$, where the insert shows character of the dispersion at resonant frequencies; (c) microwave magnetization component $m_{z}$ distribution across the sample thickness.

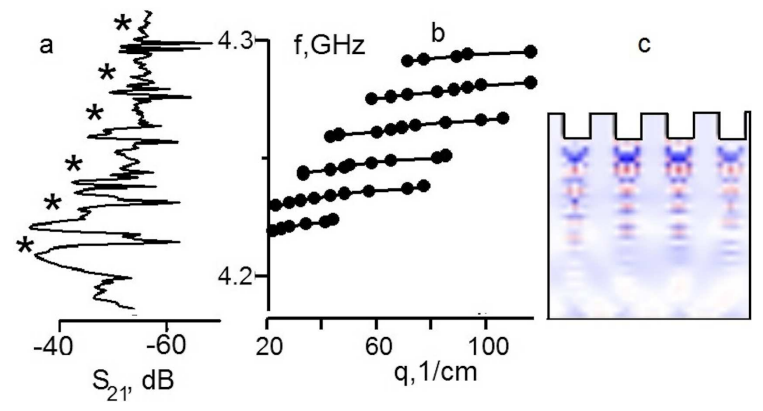

Fig. 3. Laue geometry, MSSW propagates along grooves, applied field $H \approx 820$ Oe corresponds to $\theta \approx 0$ : (a) MSSW transmission coefficients $S_{21}(f)$; (b) MSSW dispersion $f(q)$, (c) microwave magnetization component $\mathrm{mz}$ distribution across the sample thickness. All other parameters correspond to Fig. 2.

simulation using OOMMF [5] performed for microwave magnetization component $m_{z}$ at resonant frequencies of MC etched in the YIG film (see Fig. 2c and Fig. 3c).

Note that for examined YIG film with leaky MS, the nature of the interaction of the MSSW with the guided exchange modes is substantially determined by the angle $\theta$. Moreover, number of the resonances in $S_{21}(f)$ dependencies and anomalous regions in $f(q)$ was maxi- mal at $\theta \approx 0^{\circ}$ (Fig. 3) and minimal at $\theta \approx 90^{\circ}$ (Fig. 2). In the Laue geometry $\left(\theta \approx 0^{\circ}\right)$, the propagation of the MSSW in the structure with the leaky MS is accompanied by the resonant interaction with the exchange modes of the transversely magnetized subwavelength channels formed under etched grooves by static demagnetization fields (Fig. 3c). In this case, the frequencies of the observed MSSW absorption (marked by asterisks in Fig. 3a) or transmission can be related to the formation of antiBorrmann and Borrmann modes of the structure [6].

It is worth to note that in our experiments the long wavelength frequency limit $f_{0}=\gamma \sqrt{H(H+4 \pi M)}(\gamma=$ $2.8 \mathrm{MHz} / \mathrm{Oe}$ ) in MSSW spectrum was found to be practically the same at different angles $\theta: f_{0}(\theta) \approx$ const. This effect can be understood within the framework of the approach based on the effective medium model [7]. We found that for MSSW in the films with leaky MS both the dependence $f_{0}(\theta)$ and dispersion $f(q)$ (at frequencies outside the resonant frequencies) could be well described by the model of homogeneous film having internal magnetic field $\boldsymbol{H}_{i n}(\boldsymbol{r})$ equal to the internal field of the leaky MS structure averaged over the period $\Lambda$.

\subsection{Resonant $M S$}

The structure with "resonant MS" was formed by placing a subwavelength periodic array of magnetic YIG microstripes on the top of the plane YIG film with thickness $d \approx 7 \mu \mathrm{m}$, see Fig. 4 . The stripes array was formed out of $1 \mu \mathrm{m}$ thick YIG film with $4 \pi M \approx 1550 \mathrm{G}$ grown by ion beam evaporation $[8]$ and had the period $\Lambda \approx 12 \mu \mathrm{m}$ and stripes width $\approx 6 \mu \mathrm{m}$.

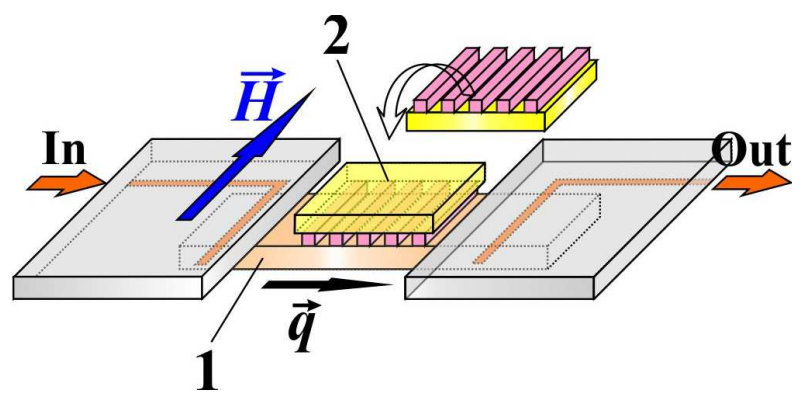

Fig. 4. YIG film with "resonant MS": 1 - plane YIG film with thickness $d \approx 7 \mu \mathrm{m} ; 2-$ YIG microstripe array with period $\Lambda \approx 12 \mu \mathrm{m}$, stripe thickness $\approx 1 \mu \mathrm{m}$ and width $\approx 6 \mu \mathrm{m}$.

Figure 5 shows measured transmission characteristics and dispersion for MSSW propagating in the plane YIG film only (curve 1) and with the stripes array placed on it (curve 2). Dispersion characteristics were plotted using experimental phase dependencies on frequency similar to the methodology described in [9]. The horizontal dashed line in Fig. 5 points out the frequency corresponding to the resonant absorption band in the transmission spectrum $S_{21}(f)$ and the anomalous part of dispersion curve $f(q)$. 
Obtained results can be explained using the approximation of effective medium [7] for description of microwave properties of YIG microstripe arrays. In this case resonant features in $S_{12}(f)$ and $f(q)$ dependencies can be treated as a result of coupling between two MSSW's propagating in layered structures: one goes through the thick plane YIG film and other one through the thin YIG film with effective parameters equivalent to the YIG microstripe array $[4,7,10]$. Curve 3 in Fig. 5 corresponds to the MSSW dispersion calculated for the YIG film with chosen parameters separated by $50 \mu \mathrm{m}$ thick air gap from the YIG film with the effective parameters corresponding to the stripes array [4]. One can see the anti-crossing effect at the resonance frequencies, that gives possibility to consider the composite structure itself as the film with the "resonant metasurface" [1].

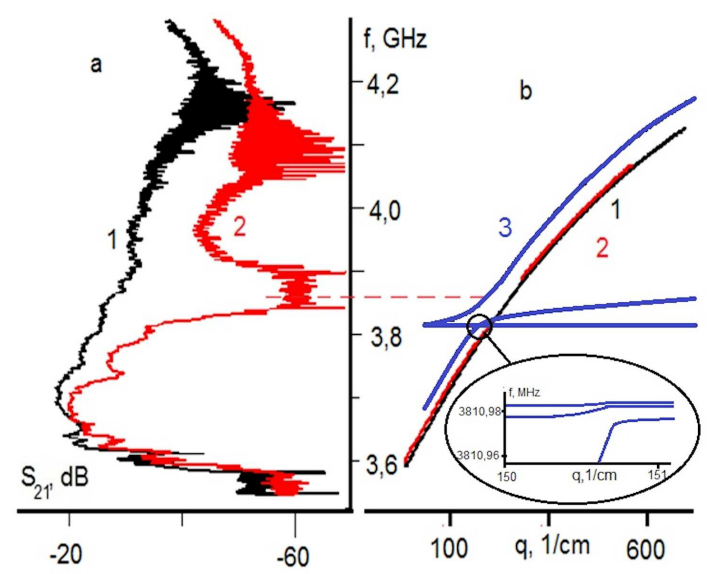

Fig. 5. MSSW propagation in the YIG film with "resonant MS" at $H \approx 650$ Oe: (a) MSSW transmission coefficients $S_{21}(f)$ in the plane film (curve 1) and in the film with "resonant MS" (curve 2); (b) 1 and 2 corresponding MSSW dispersions $f(q), 3$ - calculated dispersion. Horizontal dashed line shows the position of the resonant absorption in $S_{21}(f)$ dependence and magnonic gap in dispersion curve $f(q)$.

\section{Conclusions}

In this work we discussed the experimental results on the MSSW propagation in YIG films with "leaky" and "resonant" metasurfaces. The first type of MS was in the form of the grooves with subwavelength period etched in the YIG film surface and the second one - the plane YIG film with the subwavelength periodic array of micronsized magnetic stripes placed on its surface. Such structures demonstrated effects of MSSW filtration and formation of anomalous regions in its dispersion. Dependencies of these effects on the angle $\theta$ between the direction of applied magnetic field and the perpendicular to the grooves' axis were studied for the film with 1D "leaky" MS. Number and width of anomalous regions in the MSSW dispersion were minimal for $\theta=90^{\circ}$ and maximal for $\theta=0^{\circ}$
(Laue geometry). Such regions were explained as the resonant interaction of the MSSW with the leaky exchange modes that appeared due to demagnetization fields created by the surface periodic structure. MSSW diffraction in the Laue geometry was analogical to the formation of the Borrmann and anti-Borrmann modes in photonic crystal [6]. The long wavelength frequency limit of the MSSW spectrum was found to be practically independent on angle $\theta$. That effect and MSSW dispersion in the long-wavelength range can be described with sufficient accuracy by the model of the homogeneous film with internal magnetic field equal to the internal field of the structure with MS averaged over the period.

MSSW propagation in YIG film with "resonant MS" accompanied with the appearance of resonant features in the transmission characteristics and dispersions and can be considered as a result of the resonant interaction of two MSSW propagating in two plane YIG films. One of these films is actual thick YIG film and the other one is the effective thin YIG film that is equivalent to the YIG microstripe array.

\section{Acknowledgments}

This work was supported by the RFBR grants \# 1657-00153 Bel-a, 17-07-01452.

\section{References}

[1] S.B. Glybovski, S.A. Tretyakov, P.A. Belov, Y.S. Kivshar, C.R. Simovski, Phys. Rep. 634, 1 (2016).

[2] H.-T. Chen, A.J. Taylor, N. Yu, Rep. Prog. Phys. 79, 076401 (2016).

[3] S.L. Vysotskii, S.A. Nikitov, Y.A. Filimonov, J. Exp. Theor. Phys. 101, 547 (2005).

[4] M. Krawczyk, D. Grundler, J Phys Condens Matter. 26, 123202 (2014).

[5] M.J. Donahue, D.G. Porter, OOMMF User's Guide, Version 1.0. NISTIR 6376, National Institute of Standards and Technology, Gaithersburg, MD (Sept 1999).

[6] I.E. Razdol'skii, T.V. Murzina, O.A. Aktsipetrov, M. Inoue, JETP Lett. 87, 395 (2008).

[7] X.-Z. Wang, D.R. Tilley, Phys. Rev. B 50,13472 (1994).

[8] A.I. Stognij, L.V. Lutsev, V.E. Bursian, N.N. Novitskii, JAP, 118, 023905 (2015).

[9] Y.V. Khivintsev, Y.A. Filimonov, S.A. Nikitov, Appl. Phys. Lett., 106, 052407 (2015).

[10] M. Mruczkiewicz, M. Krawczyk, G. Gubbiotti, S. Tacchi, Yu.A. Filimonov, D.V. Kalyabin, I.V. Lisenkov, S.A. Nikitov, New J. Phys. 15, 113023 (2013). 